\title{
THE EFFECT OF SILICON FOLIAR APPLICATION ON THE DEVELOPMENT OF SEASON ORNAMENTAL PLANTS. PART II: Argyranthemum frutescens 'Blazer Rose', Xerochrysum bracteatum 'Gold', Osteospermum ecklonis 'Grande Pink Blush' AND Gaura lindheimeri 'Corinas Choice'
}

\author{
Katarzyna Wróblewska, Regina Dębicz \\ Department of Horticulture, Wroclaw University of Environmental and Life Sciences, \\ pl. Grunwladzki 24 A, 50-363 Wrocław, Poland \\ e-mail: katarzyna.wroblewska@up.wroc.pl
}

Received: 17.06.2011

\begin{abstract}
Recent research has proved that supplementary application of silicon, involved in plant tolerance against many stress factors, positively influences plant development improving the quality of ornamental plants. A two-factorial experiment involved four cultivars of ornamental plants: Argyranthemum frutescens 'Blazer Rose', Xerochrysum bracteatum 'Gold', Osteospermum ecklonis 'Grande Pink Blush', and Gaura lindheimeri 'Corinas Choice' The first experimental factor was the concentration of Actisil preparation being an equivalent of 60, 120,

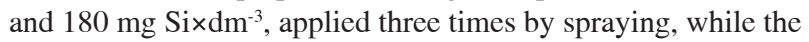
second one was the type of medium: peat substrate and peat substrate with sand. Actisil had a beneficial effect on the number of lateral shoots in all plants. The highest number of buds and inflorescences was determined in Xerochrysum and Osteospermum plants sprayed with $0.3 \%$ Actisil, and $0.2 \%$ Actisil in case of Argyranthemum and Gaura. The effect of medium type on plant growth depended on plant species. Argyranthemum and Osteospermum plants cultivated in the medium with sand showed inhibited growth, although medium type did not affect the growth of Xerochrysum.
\end{abstract}

Key words: silicon, seasonal flowering plants, growth, flowering

\section{INTRODUCTION}

Silicon, a chemical element commonly occurring in nature, has a positive effect on plant development and resistance. Its properties include the beneficial effect on the ionic balance in plants, reducing toxic effects of manganese and iron excessive amounts, as well as reinforcement of cell walls (M a r s h ner et al. 1990). This element is also known for its stimulation of plant resistance to fungal diseases ( $\mathrm{M} \mathrm{c} \mathrm{A} \mathrm{v} \mathrm{o} \mathrm{y}$ and Bible, 1996; Gill man et al. 2003), insects (R e ynolds et al. 2009), and resistance to unfavourable environmental conditions, among others, too low temperature or water deficit. One of the mechanisms for supporting plants under water stress conditions is decreased transpiration resulting in reduction in water loss, but silicon also partakes in osmoregulation, maintaining water status and adequate supply of nutrients (S a c a ł a , 2009).

Both biotic and abiotic stress factors induce physiological disorders leading to loss in yields and, in case of ornamental plants, to loss of decorative value. Although silicon is not an indispensable element for the growth and development of higher plants, according to E p s te in (2009), under stress conditions additional treatment with silicon may cause a big difference in their performance. It is particularly important in glasshouse production as it involves cultivation of plants in soiless media which do not contain silicon. Additional treatment with $\mathrm{Si}$ does not cause severe injury to plants as its excessive amounts may be secreted.

The main form of silicon, available to plants, is orthosilicic acid, absorbed by plants and precipitated throughout the plant as amorphous silica ( $\mathrm{R} \mathrm{i} \mathrm{c} \mathrm{h} \mathrm{m} \mathrm{o} \mathrm{n} \mathrm{d}$ and S u s s m a n, 2003). In case of ornamental plants, this element is also applied in the form of silicates, e.g. potassium silicate and sodium silicate as drenches or foliar applications, and it can be also added to medium mixtures, e.g. in the form of rice husk ash ( $\mathrm{F} \mathrm{r} \mathrm{a} \mathrm{n} \mathrm{t} \mathrm{z}$ et al. 2005; K a me nidou et al. 2008, 2010). 
The aim of the research was to assess the effect of orthosilicic acid contained in Actisil preparation as well as two types of soil on growth and flowering of selected decorative plants for balconies and terraces.

\section{MATERIALS AND METHODS}

In the years 2009-2010, an experiment was carried out aiming to assess the effect of Actisil preparation, containing $0.6 \%$ of silicon in the form of $\mathrm{H}_{4} \mathrm{SiO}_{4}$, on growth and development of four seasonal species: Boston daisy Argyranthemum frutescens (L.) Sch. Bip. 'Blazer Rose', paper daisy Xerochrysum bracteatum (Vent.)Tzvelev 'Gold', African daisy Osteospermum ecklonis (DC.) Norl. 'Grande Pink Blush', and Gaura lindheimeri Engelm. et A.Gray 'Corinas Choice'.

Treatments as well as data recording and analysis followed those presented in Part I of the article.

\section{RESULTS AND DISCUSSION}

The influence of silicon on plant development depends on, among others, cultivation conditions and plant species. Among higher plants, some monocotyledons, e.g. the Gramineae (Poaceae) which accumulate high amounts of silicon (more than $1 \%$ of dry mass) ( $\mathrm{M} \mathrm{a}$ and $\mathrm{Yam}$ a j i 2008), show a good response to silicon treatment, whereas Dicotyledones are believed to be non-accumulators of Si. For this reason, monocots have become the main object of silicon research, with special focus on rice. Apart from these observations, some dicotyledons, like Aster, are beneficially affected by Si treatment (F r a n t z et al. 2005).

One of the criteria of the plant growth rate is their height. It has been assumed that more intensive development produce plants of increased height. Yet, in the case of numerous ornamentals, taller plants are not the most desired ones. Compact plants showing considerable tillering are often regarded as the most attractive. This requirement is especially important in the case of pot-grown ornamentals. According to $\mathrm{Startek}$ et al. (2006), silicon contained in Actisil preparation improves plant habit of pot-grown chrysanthemums, stimulating shoot elongation of varieties characterized by moderate growth and inhibiting excessive extension of shoots in varieties showing such a tendency. It also improves the quality of garden pansy Viola $\mathrm{x}$ wittrockiana (W rag a and Dobrow ols ka, 2007).

Abundant flowering is extremely important for plants with decorative flowers, which results, among others, from the degree of tillering. One of the most significant factors stimulating lateral bud development is the cytokinin level in shoots. It was detected that foliar treatment with silicon could increase the level of cytokinin in wheat plants ( $\mathrm{H}$ a n a f y A h med et al. 2008). In both years of our experiment, we proved the beneficial effect of orthosilicic acid contained in Actisil preparation on the number of lateral shoots in all plant species selected for the experiment. Irrespective of type of medium and year of experiment, the higher was the concentration of Actisil, the better tillering of Argyranthemum (Table 1) and Xerochrysum plants (Table 2) was found.

In Osteospermum, the two highest concentrations proved to be the most desired ones - they strongly affected, in the same way, the number of lateral shoots (Table 3) - while in Gaura the highest number of lateral shoots was produced due to spraying with Actisil of $0.2 \%$ concentration (Table 4 ). As its concentration increased, this preparation more evidently affected lateral shoot elongation in paper daisy and Gaura. In the case of Argyranthemum, longer lateral shoots were recorded in plants sprayed with higher concentrations of Actisil, while in Osteospermum only $0.3 \%$ solution of this preparation resulted in shoot elongation (Table 3).

The number of African daisy and paper daisy inflorescences increased according to the increase in concentration of fertilizer solution and it was connected with longer shoots. In the same taxa, Actisil extended the diameter of inflorescences (Fig. 1). This finding confirms the research results obtained so far, as the beneficial effect of silicon on inflorescence diameter has been proved in case of some species of the same family Asteraceae, such as sunflower Helianthus annuus L. (K a menidou et al. 2008; Carvalho et al. 2009), zinnia Zinnia (K a m e n i d o u et al. 2009), and gerbera Gerbera (S a v v a s et al. 2002; K a m e $\mathrm{nid} \mathrm{ou} \mathrm{et} \mathrm{al.} \mathrm{2010).} \mathrm{Regardless} \mathrm{of} \mathrm{its} \mathrm{concentration,}$ this preparation stimulated flowering of Gaura, yet the highest number of flowers was produced by plants treated with $0.2 \%$ solution of Actisil (Fig.2). The preparation at the same concentration had a beneficial effect on the number and diameter of Argyranthemum inflorescences. On the other hand, at a concentration of $0.1 \%$ Actisil inhibited flowering of this variety. These two taxa had larger flowers only under the influence of the highest concentration of the preparation $(0.3 \%)$.

Actisil significantly regulated the height of such plants as Xerochrysum and Gaura. This parameter showed higher values according to the increase in Actisil concentration. Under the influence of Actisil, higher plants were also characterized by a larger plant diameter. Similar results, regarding the application of Actisil, also introduced three times, were obtained for multiflora rose Rosa multiflora Thunb. ex Murray (H e tman et al. 2007). Boston daisy sprayed with Actisil at a concentration of $0.3 \%$ reached a 1.7 times larger diameter. A similar phenomenon - an increased diameter of plants obtained by treatment with $0.2 \%$ Actisil - was observed in garden pansy (W r a g a and Dobrowolska, 2007). Osteospermum showed 
only a slight influence of this preparation as far as plant height and diameter were concerned.

Plant growth also depended on the type of medium the plants were grown in. In Argyranthemum and Osteospermum, inhibited growth characterized plants cultivated in medium with the addition of sand. It was manifested in decreased plant height and diameter values; Argyranthemum plants also showed insufficient tillering as well as poorer flowering (Fig. 4). However, the type of medium did not affect vegetative development of paper daisy, except for plant height which increased in plants grown in peat substrate. Since plant height is not a decisive characteristic for the quality of Xerochrysum as a container-grown plant, it can be well cultivated using cheaper medium with the addition of sand. Gaura, growing in a mixture of peat substrate and sand, demonstrated decreased plant diameter and less numerous, but longer lateral shoots. Longer shoots of this taxon produced higher number of flowers (Fig. 4). The type of growing medium did not influence flower and inflorescence diameter (Fig. 3) in none of the examined plant varieties.

Chlorophyll content in plants affects the rate of photosynthesis, thus, indirectly, plant growth. Yet, no effect was recorded of Actisil on chlorophyll content in leaves of strawberry Fragaria ananassa Duch. of the 'Elvira' variety, nor it influenced the rate of $\mathrm{CO}_{2}$ assimilation, although plants treated with silicon produce higher quantities of biomass (Mikiciuk and Mikiciuk, 2009). According to Startek et al. (2006), the greening index of chrysanthemum leaves, correlated with chlorophyll content in leaves, depended on Actisil concentration and dose, as well as on variety and time of fertilizer application. Similar results were obtained in our investigations, where Actisil increased chlorophyll content in leaves of Osteospermum. Increased chlorophyll content was found in Argyranthemum plants treated with Actisil at 0.1 and 0.2 concentrations as well as in Gaura treated with Actisil at a 0.1 concentration. In all concentrations, Actisil increased chlorophyll content in Gau$r a$ grown in the mixture of peat substrate and sand (Table 5). Actisil, however, had a negative effect on chlorophyll content in Xerochrysum leaves, nevertheless, that effect was weaker in plants cultivated in the medium containing sand. The lower content of chlorophyll in this species was not translated into its growth intensity.

Table 1.

Morphological features of Argyranthemum frutescens 'Blazer Rose'

depending on Actisil concentration and type of medium

\begin{tabular}{|c|c|c|c|c|c|c|c|c|c|c|c|c|c|}
\hline \multirow{4}{*}{$\begin{array}{c}\text { Concentration } \\
{[\%]} \\
\text { (C) }\end{array}$} & \multirow{4}{*}{$\begin{array}{c}\text { Year } \\
\text { A }\end{array}$} & \multicolumn{12}{|c|}{ Feature of the plant } \\
\hline & & \multirow{2}{*}{\multicolumn{3}{|c|}{$\begin{array}{l}\text { Height of plants }[\mathrm{cm}] \\
\text { Type of medium (B) }\end{array}$}} & \multirow{2}{*}{\multicolumn{3}{|c|}{$\frac{\text { Diameter of plants }[\mathrm{cm}]}{\text { Type of medium }(\mathrm{B})}$}} & \multirow{2}{*}{\multicolumn{3}{|c|}{$\begin{array}{c}\text { Number of lateral shoots } \\
\text { Type of medium (B) }\end{array}$}} & \multirow{2}{*}{\multicolumn{3}{|c|}{$\begin{array}{c}\text { Length of lateral shoots [cm } \\
\text { Type of medium (B) }\end{array}$}} \\
\hline & & & & & & & & & & & & & \\
\hline & & $\mathrm{P}^{*}$ & PS* & $\begin{array}{l}\text { Mean } \\
(\mathrm{AxC})\end{array}$ & $\mathrm{P}^{*}$ & PS* & $\begin{array}{l}\text { Mean } \\
(\mathrm{AxC})\end{array}$ & $\mathrm{P}^{*}$ & PS* & $\begin{array}{l}\text { Mean } \\
(\mathrm{AxC})\end{array}$ & $\mathrm{P}^{*}$ & PS* & $\begin{array}{l}\text { Mean } \\
(\mathrm{AxC})\end{array}$ \\
\hline \multirow{2}{*}{ Control } & 2009 & 35.2 & 34.0 & 34.6 & 12.8 & 10.7 & 11.8 & 7.3 & 4.2 & 5.8 & 12.4 & 5.5 & 9.0 \\
\hline & 2010 & 12.0 & 10.5 & 11.3 & 12.9 & 12.3 & 12.6 & 6.9 & 5.5 & 6.2 & 10.0 & 7.7 & 8.9 \\
\hline \multicolumn{2}{|l|}{ Mean $(\mathrm{BxC})$} & 23.6 & 22.3 & 22.9 & 12.9 & 11.5 & 12.2 & 7.1 & 4.9 & 6.0 & 11.2 & 6.6 & 8.9 \\
\hline \multirow{2}{*}{$0.1 \%$} & 2009 & 29.7 & 23.5 & 26.6 & 17.0 & 15.4 & 16.2 & 8.0 & 7.5 & 7.7 & 8.5 & 7.4 & 8.0 \\
\hline & 2010 & 14.9 & 11.2 & 13.1 & 17.5 & 15.0 & 16.3 & 8.5 & 6.4 & 7.5 & 11.0 & 8.0 & 9.5 \\
\hline \multicolumn{2}{|l|}{ Mean $(\mathrm{BxC})$} & 22.3 & 17.4 & 19.8 & 17.3 & 15.2 & 16.3 & 8.3 & 7.0 & 7.6 & 9.8 & 7.7 & 8.8 \\
\hline \multirow{2}{*}{$0.2 \%$} & 2009 & 37.4 & 30.8 & 34.1 & 20.6 & 16.8 & 18.7 & 8.5 & 6.4 & 7.5 & 12.1 & 8.6 & 10.4 \\
\hline & 2010 & 16.5 & 13.9 & 15.2 & 21.4 & 16.9 & 19.2 & 8.9 & 8.2 & 8.6 & 13.5 & 8.7 & 11.1 \\
\hline \multicolumn{2}{|l|}{ Mean $(\mathrm{BxC})$} & 27.0 & 22.4 & 24.7 & 21.0 & 16.9 & 19.0 & 8.7 & 7.3 & 8.0 & 12.8 & 8.7 & 10.8 \\
\hline \multirow{2}{*}{$0.3 \%$} & 2009 & 34.5 & 27.5 & 31.0 & 21.7 & 18.7 & 20.2 & 13.5 & 9.6 & 11.6 & 12.6 & 9.6 & 11.1 \\
\hline & 2010 & 29.9 & 24.9 & 27.4 & 24.8 & 18.0 & 21.4 & 15.0 & 10.0 & 12.5 & 15.0 & 10.9 & 13.0 \\
\hline \multicolumn{2}{|l|}{ Mean $(\mathrm{BxC})$} & 32.2 & 26.2 & 29.2 & 23.3 & 18.4 & 20.8 & 14.3 & 9.8 & 12.1 & 13.8 & 10.3 & 12.1 \\
\hline \multicolumn{2}{|l|}{ Mean (B) } & 26.3 & 22.1 & & 18.6 & 15.5 & & 9.6 & 7.3 & & 11.9 & 8.3 & \\
\hline \multicolumn{2}{|l|}{$\mathrm{LSD}_{0.05} ; \mathrm{NIR}_{0.05}$} & \multicolumn{3}{|c|}{$\begin{aligned} \mathrm{B} & =0.6 \\
\mathrm{C} & =0.9 \\
\mathrm{xC} & =1.2 \\
\mathrm{xC} & =1.2\end{aligned}$} & \multicolumn{3}{|c|}{$\begin{aligned} \mathrm{B} & =0.9 \\
\mathrm{C} & =1.3 \\
\mathrm{xC} & =\text { n.s.; r.n. } \\
\mathrm{xC} & =1.9\end{aligned}$} & & $\begin{array}{l}\mathrm{B}=0.6 \\
\mathrm{C}=0.8 \\
\mathrm{C}=1.1 \\
\mathrm{x}=\text { r.n. }\end{array}$ & ; n.s. & & $\begin{aligned} \mathrm{B} & =0.4 \\
\mathrm{C} & =0.6 \\
\mathrm{X} & =0.9 \\
\mathrm{x} & =0.9\end{aligned}$ & \\
\hline
\end{tabular}

* P means peat substrate, PS means peat substrate with sand in 3:1 ratio 
Table 2.

Morphological features of Xerochrysum bracteatum 'Gold' depending on Actisil concentration and type of medium

\begin{tabular}{|c|c|c|c|c|c|c|c|c|c|c|c|c|c|}
\hline \multirow{4}{*}{$\begin{array}{c}\text { Concentration } \\
{[\%]} \\
\text { (C) }\end{array}$} & \multirow{4}{*}{$\begin{array}{l}\text { Year } \\
(\mathrm{A})\end{array}$} & \multicolumn{12}{|c|}{ Feature of the plant } \\
\hline & & \multirow{2}{*}{\multicolumn{3}{|c|}{$\begin{array}{c}\text { Height of plants }[\mathrm{cm}] \\
\text { Type of medium }(\mathrm{B})\end{array}$}} & \multirow{2}{*}{\multicolumn{3}{|c|}{$\begin{array}{c}\text { Diameter of plants [cm] } \\
\text { Type of medium }(\mathrm{B})\end{array}$}} & \multicolumn{6}{|c|}{ Number of lateral shoots Length of lateral shoots $[\mathrm{cm}]$} \\
\hline & & & & & & & & \multicolumn{3}{|c|}{ Type of medium (B) } & \multicolumn{3}{|c|}{ Type of medium (B) } \\
\hline & & $\mathrm{P} *$ & PS* & $\begin{array}{l}\text { Mean } \\
(\mathrm{AxC})\end{array}$ & $\mathrm{P}^{*}$ & PS* & $\begin{array}{l}\text { Mean } \\
(\mathrm{AxC})\end{array}$ & $\mathrm{P}^{*}$ & PS* & $\begin{array}{l}\text { Mean } \\
(\mathrm{AxC})\end{array}$ & $\mathrm{P}^{*}$ & PS* & $\begin{array}{l}\text { Mean } \\
(\mathrm{AxC})\end{array}$ \\
\hline \multirow{2}{*}{ Control } & 2009 & 16.6 & 14.9 & 15.8 & 15.0 & 15.8 & 15.4 & 4.2 & 4.4 & 4.3 & 10.4 & 10.3 & 10.4 \\
\hline & 2010 & 13.9 & 11.9 & 12.9 & 13.5 & 13.9 & 13.7 & 4.7 & 4.3 & 4.5 & 9.5 & 8.8 & 9.2 \\
\hline Mean $(\mathrm{BxC})$ & & 15.3 & 13.4 & 14.3 & 14.3 & 14.9 & 14.6 & 4.5 & 4.4 & 4.4 & 10.0 & 9.6 & 9.8 \\
\hline \multirow{2}{*}{$0.1 \%$} & 2009 & 17.0 & 15.3 & 16.2 & 15.3 & 16.2 & 15.8 & 4.4 & 5.0 & 4.7 & 12.5 & 13.2 & 12.9 \\
\hline & 2010 & 14.3 & 12.5 & 13.4 & 15.7 & 14.5 & 15.1 & 5.2 & 4.7 & 5.0 & 12.5 & 11.9 & 12.2 \\
\hline Mean $(\mathrm{BxC})$ & & 15.7 & 13.9 & 14.8 & 15.5 & 15.4 & 15.5 & 4.8 & 4.9 & 4.9 & 12.5 & 12.6 & 12.6 \\
\hline \multirow{2}{*}{$0.2 \%$} & 2009 & 20.9 & 17.2 & 19.1 & 16.2 & 17.6 & 16.9 & 5.4 & 5.7 & 5.6 & 14.7 & 15.2 & 15.0 \\
\hline & 2010 & 15.5 & 13.9 & 14.7 & 16.4 & 16.5 & 16.5 & 5.6 & 5.2 & 5.4 & 16.5 & 15.2 & 15.9 \\
\hline Mean $(\mathrm{BxC})$ & & 18.2 & 15.6 & 16.9 & 16.3 & 17.1 & 16.7 & 5.5 & 5.5 & 5.5 & 15.6 & 15.2 & 15.4 \\
\hline \multirow{2}{*}{$0.3 \%$} & 2009 & 20.0 & 20.1 & 20.1 & 19.0 & 19.6 & 19.3 & 5.8 & 7.2 & 6.5 & 16.7 & 17.6 & 17.2 \\
\hline & 2010 & 17.5 & 16.5 & 17.0 & 19.5 & 18.5 & 19.0 & 5.8 & 5.5 & 5.7 & 17.9 & 16.0 & 17.0 \\
\hline Mean $(\mathrm{BxC})$ & & 18.8 & 18.3 & 18.5 & 19.3 & 19.1 & 19.2 & 5.8 & 6.4 & 6.1 & 17.3 & 16.8 & 17.1 \\
\hline Mean (B) & & 17.0 & 15.3 & & 16.4 & 16.6 & & 5.2 & 5.3 & & 13.9 & 13.6 & \\
\hline \multicolumn{2}{|l|}{$\operatorname{LSD}_{0.05} ; \mathrm{NIR}_{0.05}$} & \multicolumn{3}{|c|}{$\begin{aligned} \mathrm{B} & =0.3 \\
\mathrm{C} & =0.4 \\
\mathrm{AxC} & =0.6 \\
\mathrm{BxC} & =0.6\end{aligned}$} & \multicolumn{3}{|c|}{$\begin{aligned} \mathrm{B} & =\text { r.n. } ; \text { n.s } \\
\mathrm{C} & =0.5 \\
\mathrm{xC} & =0.7 \\
\mathrm{xC} & =\text { n.s.; r.n. }\end{aligned}$} & \multicolumn{3}{|c|}{$\begin{aligned} \mathrm{B} & =\text { n.s.; r.n. } \\
\mathrm{C} & =0.8 \\
\mathrm{xC} & =1.2 \\
\mathrm{xC} & =\text { r.n.; n.s. }\end{aligned}$} & \multicolumn{3}{|c|}{$\begin{aligned} \mathrm{B} & =\text { n.s.; r.n. } \\
\mathrm{C} & =0.8 \\
\mathrm{AxC} & =1.2 \\
\mathrm{BxC} & =\text { n.s.; r.n. }\end{aligned}$} \\
\hline
\end{tabular}

* $\mathrm{P}$ means peat substrate, PS means peat substrate with sand in 3:1 ratio

Table 3.

Morphological features of Osteospermum ecklonis ‘Grande Pink Blush' depending on Actisil concentration and type of medium

\begin{tabular}{|c|c|c|c|c|c|c|c|c|c|c|c|c|c|}
\hline \multirow{4}{*}{$\begin{array}{c}\text { Concentration } \\
{[\%]} \\
\text { (C) }\end{array}$} & \multirow{4}{*}{$\begin{array}{l}\text { Year } \\
(\mathrm{A})\end{array}$} & \multicolumn{12}{|c|}{ Feature of the plant } \\
\hline & & \multirow{2}{*}{\multicolumn{3}{|c|}{$\begin{array}{c}\text { Height of plants }[\mathrm{cm}] \\
\text { Type of medium (B) }\end{array}$}} & \multirow{2}{*}{\multicolumn{3}{|c|}{$\begin{array}{c}\text { Diameter of plants }[\mathrm{cm}] \\
\text { Type of medium }(\mathrm{B})\end{array}$}} & \multicolumn{6}{|c|}{ Number of lateral shoots Length of lateral shoots [cm } \\
\hline & & & & & & & & \multicolumn{3}{|c|}{ Type of medium (B) } & \multicolumn{3}{|c|}{ Type of medium (B) } \\
\hline & & $\mathrm{P}^{*}$ & PS* & $\begin{array}{l}\text { Mean } \\
(\mathrm{AxC})\end{array}$ & $\mathrm{P}^{*}$ & PS* & $\begin{array}{l}\text { Mean } \\
(\mathrm{AxC})\end{array}$ & $\mathrm{P}^{*}$ & PS* & $\begin{array}{l}\text { Mean } \\
(\mathrm{AxC})\end{array}$ & $\mathrm{P}^{*}$ & PS* & $\begin{array}{l}\text { Mean } \\
(\mathrm{AxC})\end{array}$ \\
\hline \multirow{2}{*}{ Control } & 2009 & 48.2 & 47.8 & 48.0 & 27.6 & 21.5 & 24.6 & 6.7 & 5.3 & 6.0 & 21.6 & 19.4 & 20.5 \\
\hline & 2010 & 36.9 & 35.6 & 36.3 & 20.5 & 20.0 & 20.3 & 4.5 & 4.0 & 4.3 & 11.7 & 10.8 & 11.3 \\
\hline Mean $(\mathrm{BxC})$ & & 42.6 & 41.7 & 42.2 & 24.1 & 20.8 & 22.5 & 5.6 & 6.7 & 5.1 & 16.7 & 15.1 & 15.9 \\
\hline \multirow{2}{*}{$0.1 \%$} & 2009 & 44.6 & 48.9 & 46.8 & 21.3 & 21.8 & 21.6 & 6.6 & 6.2 & 6.4 & 15.9 & 14.4 & 15.2 \\
\hline & 2010 & 40.2 & 39.7 & 40.0 & 20.9 & 20.5 & 20.7 & 5.3 & 5.0 & 5.2 & 12.6 & 12.0 & 12.3 \\
\hline Mean $(\mathrm{BxC})$ & & 42.4 & 44.3 & 43.4 & 21.1 & 21.2 & 21.2 & 5.9 & 5.6 & 5.8 & 14.3 & 13.2 & 13.8 \\
\hline \multirow{2}{*}{$0.2 \%$} & 2009 & 45.5 & 47.3 & 46.4 & 23.3 & 23.1 & 23.2 & 8.7 & 9.2 & 9.0 & 17.6 & 16.8 & 17.1 \\
\hline & 2010 & 43.6 & 42.3 & 43.0 & 23.4 & 22.3 & 22.9 & 5.5 & 5.8 & 5.6 & 14.3 & 13.6 & 13.9 \\
\hline Mean $(\mathrm{BxC})$ & & 44.6 & 44.8 & 44.7 & 23.4 & 22.7 & 23.1 & 7.1 & 7.5 & 7.3 & 15.9 & 15.2 & 15.5 \\
\hline \multirow{2}{*}{$0.3 \%$} & 2009 & 40.5 & 35.9 & 38.2 & 25.0 & 25.0 & 25.0 & 9.0 & 8.0 & 8.5 & 19.6 & 19.0 & 19.3 \\
\hline & 2010 & 46.7 & 45.9 & 46.3 & 26.8 & 25.2 & 26.0 & 6.0 & 6.4 & 6.2 & 15.7 & 14.0 & 14.9 \\
\hline Mean $(\mathrm{BxC})$ & & 43.6 & 40.9 & 42.3 & 25.9 & 25.1 & 25.5 & 7.5 & 7.2 & 7.4 & 17.6 & 16.5 & 17.0 \\
\hline Mean (B) & & 43.3 & 43.0 & & 23.6 & 22.5 & & 6.5 & 6.8 & & 16.1 & 15.0 & \\
\hline \multicolumn{2}{|l|}{$\mathrm{LSD}_{0.05} ; \mathrm{NIR}_{0.05}$} & \multicolumn{3}{|c|}{$\begin{aligned} B & =0.3 \\
C & =0.4 \\
A x C & =0.6 \\
B x C & =0.6\end{aligned}$} & \multicolumn{3}{|c|}{$\begin{aligned} B & =0.3 \\
C & =0.5 \\
A x C & =0.7 \\
3 x C & =0.7\end{aligned}$} & \multicolumn{3}{|c|}{$\begin{aligned} \mathrm{B} & =0.2 \\
\mathrm{C} & =0.3 \\
\mathrm{xC} & =0.4 \\
\mathrm{xC} & =0.4\end{aligned}$} & \multicolumn{3}{|c|}{$\begin{aligned} \mathrm{B} & =0.4 \\
\mathrm{C} & =0.6 \\
\mathrm{AxC} & =0.8 \\
\mathrm{BxC} & =\text { n.s.; }\end{aligned}$} \\
\hline
\end{tabular}

* $\mathrm{P}$ means peat substrate, PS means peat substrate with sand in 3:1 ratio 
The effect of silicon foliar application on the development of season ornamental plants. Part II: Argyranthemum frutescens... 111

Table 4.

Morphological features of Gaura lindheimeri 'Corinas Choice' depending on Actisil concentration and type of medium

\begin{tabular}{|c|c|c|c|c|c|c|c|c|c|c|c|c|c|}
\hline \multirow{4}{*}{$\begin{array}{l}\text { Concentration } \\
\qquad \%] \\
\text { (C) }\end{array}$} & \multirow{4}{*}{$\begin{array}{l}\text { Year } \\
(\mathrm{A})\end{array}$} & \multicolumn{12}{|c|}{ Feature of the plant } \\
\hline & & \multirow{2}{*}{\multicolumn{3}{|c|}{$\begin{array}{c}\text { Height of plants }[\mathrm{cm}] \\
\text { Type of medium (B) }\end{array}$}} & \multirow{2}{*}{\multicolumn{3}{|c|}{$\begin{array}{c}\text { Diameter of plants [cm] } \\
\text { Type of medium }(\mathrm{B}) \\
\end{array}$}} & \multirow{2}{*}{\multicolumn{3}{|c|}{$\begin{array}{c}\text { Number of lateral shoots } \\
\text { Type of medium (B) }\end{array}$}} & \multicolumn{3}{|c|}{ Length of lateral shoots $[\mathrm{cm}]$} \\
\hline & & & & & & & & & & & \multicolumn{3}{|c|}{ Type of medium (B) } \\
\hline & & $\mathrm{P}^{*}$ & PS* & $\begin{array}{l}\text { Mean } \\
(\mathrm{AxC})\end{array}$ & $\mathrm{P}^{*}$ & PS* & $\begin{array}{l}\text { Mean } \\
(\mathrm{AxC})\end{array}$ & $\mathrm{P}^{*}$ & PS* & $\begin{array}{l}\text { Mean } \\
(\mathrm{AxC})\end{array}$ & $\mathrm{P}^{*}$ & PS* & $\begin{array}{l}\text { Mean } \\
(\mathrm{AxC})\end{array}$ \\
\hline \multirow{2}{*}{ Control } & 2009 & 18.9 & 16.3 & 17.6 & 18.8 & 16.9 & 17.9 & 4.7 & 5.6 & 5.2 & 18.9 & 23.1 & 21.0 \\
\hline & 2010 & 16.5 & 15.0 & 15.8 & 14.3 & 12.5 & 13.4 & 3.4 & 3.0 & 3.2 & 11.1 & 10.2 & 10.7 \\
\hline Mean $(\mathrm{BxC})$ & & 17.7 & 15.7 & 16.7 & 16.6 & 14.7 & 15.7 & 4.1 & 4.3 & 4.2 & 15.0 & 16.7 & 15.9 \\
\hline \multirow{2}{*}{$0.1 \%$} & 2009 & 18.0 & 17.9 & 18.0 & 18.9 & 18.0 & 18.5 & 6.8 & 6.1 & 6.5 & 18.8 & 24.0 & 21.4 \\
\hline & 2010 & 17.3 & 16.9 & 17.1 & 15.0 & 10.2 & 12.6 & 4.7 & 4.4 & 4.6 & 12.9 & 11.9 & 12.4 \\
\hline Mean (BxC) & & 17.7 & 17.4 & 17.6 & 17.0 & 14.1 & 15.6 & 5.8 & 5.3 & 5.6 & 15.9 & 18.0 & 16.9 \\
\hline \multirow{2}{*}{$0.2 \%$} & 2009 & 22.0 & 21.8 & 21,9 & 23.4 & 21.9 & 22.7 & 9.6 & 7.0 & 8.3 & 23.9 & 27.8 & 25.9 \\
\hline & 2010 & 18.0 & 18.0 & 18.0 & 16.6 & 16.2 & 16.4 & 5.8 & 4.8 & 5.3 & 14.4 & 13.8 & 14.1 \\
\hline Mean $(\mathrm{BxC})$ & & 20.0 & 19.9 & 20.0 & 20.0 & 19.1 & 19.6 & 7.7 & 5.9 & 6.8 & 19.2 & 20.8 & 20.0 \\
\hline \multirow{2}{*}{$0.3 \%$} & 2009 & 21.7 & 21.9 & 21.8 & 27.1 & 23.5 & 25.3 & 6.3 & 5.7 & 6.0 & 30.2 & 34.4 & 32.3 \\
\hline & 2010 & 18.9 & 20.0 & 19.5 & 19.0 & 18.3 & 18.7 & 6.4 & 5.8 & 6.1 & 17.3 & 16.7 & 17.0 \\
\hline Mean (BxC) & & 20.3 & 21.0 & 20.7 & 23.0 & 20.9 & 22.0 & 6.4 & 5.8 & 6.1 & 23.7 & 25.6 & 24.7 \\
\hline Mean (B) & & 18.9 & 18.5 & & 19.2 & 17.2 & & 6.0 & 5.3 & & 18.5 & 20.3 & \\
\hline $\mathrm{LSD}_{0.05} ; \mathrm{NIR}_{0.05}$ & & \multicolumn{3}{|c|}{$\begin{aligned} \mathrm{B} & =\text { n.s.; r.n. } \\
\mathrm{C} & =0.7 \\
\mathrm{xC} & =1.0 \\
\mathrm{xC} & =1.0\end{aligned}$} & \multicolumn{3}{|c|}{$\begin{aligned} \mathrm{B} & =1.2 \\
\mathrm{C} & =1.7 \\
\mathrm{xC} & =\text { n.s.; r.n. } \\
\mathrm{xC} & =\text { n.s.; r.n. }\end{aligned}$} & \multicolumn{3}{|c|}{$\begin{aligned} \mathrm{B} & =0.2 \\
\mathrm{C} & =0.3 \\
\mathrm{xC} & =0.5 \\
\mathrm{xC} & =0.5\end{aligned}$} & \multicolumn{3}{|c|}{$\begin{aligned} \mathrm{B} & =0.5 \\
\mathrm{C} & =0.5 \\
\mathrm{AxC} & =0.7 \\
\mathrm{BxC} & =0.7\end{aligned}$} \\
\hline
\end{tabular}

* P means peat substrate, PS means peat substrate with sand in 3:1 ratio

Table 5.

Chlorophyll content in leaves of Argyranthemum frutescens 'Blazer Rose', Xerochrysum bracteatum 'Gold',

Osteospermum ecklonis 'Grande Pink Blush' and Gaura lindheimeri 'Corinas Choice' depending on Actisil concentration and type of medium, expressed as the sum of chlorophyll a and $\mathrm{b}\left[\mathrm{mg} \times \mathrm{g}^{-1}\right.$ of fresh weight]

\begin{tabular}{|c|c|c|c|c|c|c|c|c|c|c|c|c|c|}
\hline \multirow{4}{*}{$\begin{array}{c}\text { Concentration } \\
{[\%]}\end{array}$} & \multirow{4}{*}{ Year } & \multicolumn{12}{|c|}{ Species and variety } \\
\hline & & \multicolumn{3}{|c|}{ Argyranthemum frutescens } & \multicolumn{3}{|c|}{ Xerochrysum bracteatum } & \multicolumn{3}{|c|}{ Osteospermum ecklonis } & \multicolumn{3}{|c|}{ Gaura lindheimera } \\
\hline & & \multicolumn{3}{|c|}{ Type of medium } & \multicolumn{3}{|c|}{ Type of medium } & \multicolumn{3}{|c|}{ Type of medium } & \multicolumn{3}{|c|}{ Type of medium } \\
\hline & & $\mathrm{P}^{*}$ & PS* & Mean & $\mathrm{P} *$ & PS* & Mean & $\mathrm{P}^{*}$ & PS* & Mean & $\mathrm{P}^{*}$ & PS* & Mean \\
\hline \multirow{2}{*}{ Control } & 2009 & 0.82 & 0.86 & 0.84 & 1.01 & 1.08 & 1.05 & 0.97 & 1.29 & 1.13 & 1.00 & 1.25 & 1.13 \\
\hline & 2010 & 0.91 & 1.02 & 0.97 & 1.81 & 1.76 & 1.79 & 0.89 & 0.90 & 0.90 & 1.61 & 1.01 & 1.31 \\
\hline Mean & & 0.87 & 0.94 & 0.91 & 1.41 & 1.42 & 1.42 & 0.93 & 1.10 & 1.02 & 1.31 & 1.13 & 1.21 \\
\hline \multirow{2}{*}{0.1} & 2009 & 0.94 & 0.65 & 0.80 & 0.89 & 0.99 & 0.94 & 1.02 & 1.01 & 1.02 & 1.11 & 1.24 & 1.18 \\
\hline & 2010 & 1.31 & 1.22 & 1.27 & 1.06 & 1.42 & 1.24 & 1.20 & 1.23 & 1.22 & 1.42 & 1.78 & 1.60 \\
\hline Mean & & 1.13 & 0.94 & 1.04 & 0.98 & 1.21 & 1.10 & 1.11 & 1.12 & 1.12 & 1.27 & 1.51 & 1.39 \\
\hline \multirow{2}{*}{0.2} & 2009 & 0.75 & 0.84 & 0.80 & 0.61 & 0.79 & 0.70 & 1.11 & 1.26 & 1.19 & 0.79 & 1.17 & 0.98 \\
\hline & 2010 & 1.34 & 1.41 & 1.43 & 0.89 & 1.63 & 1.26 & 1.07 & 1.02 & 1.04 & 1.12 & 1.22 & 1.17 \\
\hline Mean & & 1.05 & 1.13 & 1.09 & 0.75 & 1.21 & 0.98 & 1.09 & 1.14 & 1.12 & 0.96 & 1.20 & 1.08 \\
\hline \multirow{2}{*}{0.3} & 2009 & 0.82 & 0.66 & 0.74 & 1.10 & 1.13 & 1.12 & 0.93 & 1.38 & 1.16 & 0.87 & 1.12 & 1.00 \\
\hline & 2010 & 1.10 & 1.01 & 1.06 & 1.02 & 1.58 & 1.30 & 1.03 & 0.96 & 1.00 & 1.27 & 1.39 & 1.33 \\
\hline Mean & & 0.96 & 0.84 & 0.90 & 1.06 & 1.36 & 1.21 & 0.98 & 1.17 & 1.08 & 1.07 & 1.26 & 1.17 \\
\hline
\end{tabular}

* P means peat substrate, PS means peat substrate with sand in 3:1 ratio 


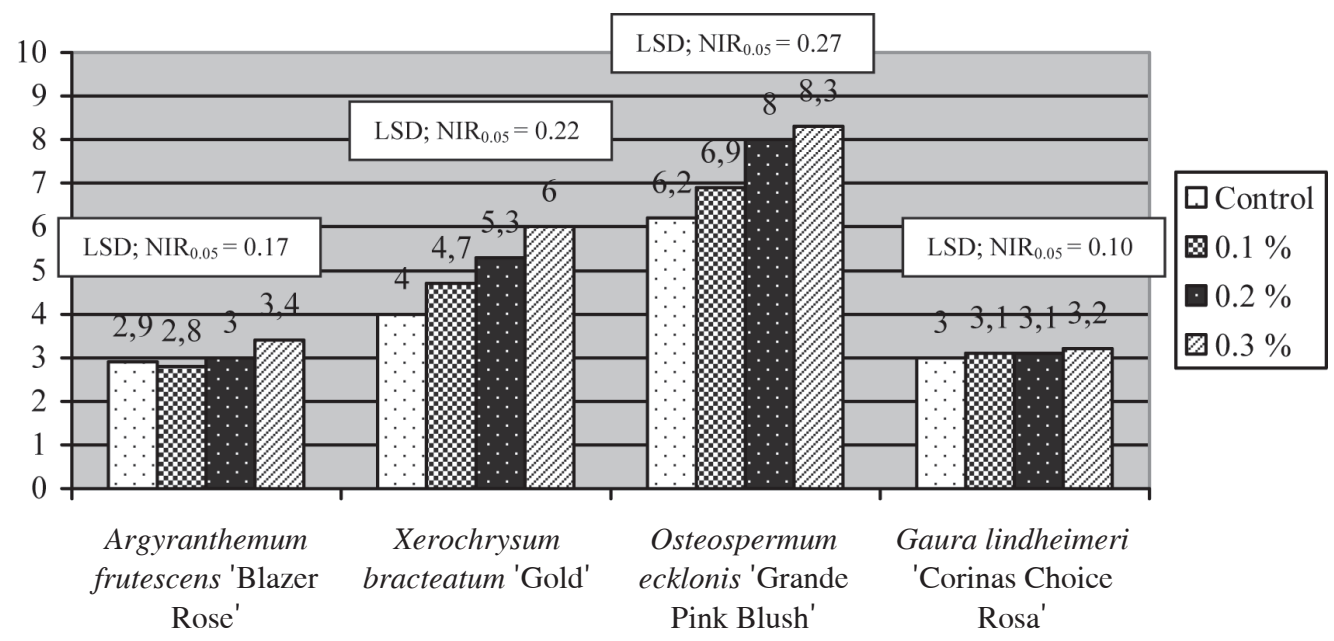

Fig. 1. The effect of Actisil concentration on the diameter of inflorescences of Argyranthemum frutescens 'Blazer Rose', Xerochrysum bracteatum 'Gold' and Osteospermum ecklonis 'Grande Pink Blush' as well as flowers of Gaura lindheimeri 'Corinas Choice' (means for two years) [cm]

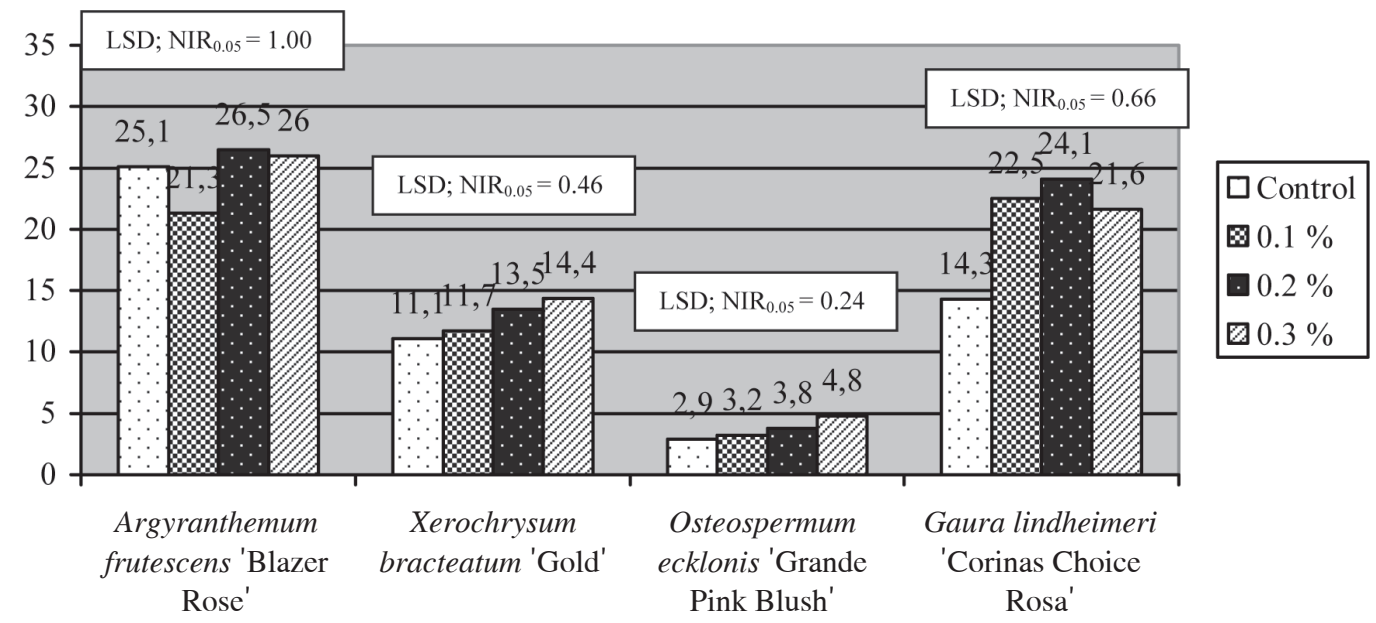

Fig. 2. The effect of Actisil concentration on the number of buds and inflorescences of Argyranthemum frutescens 'Blazer Rose', Xerochrysum bracteatum 'Gold', and Osteospermum ecklonis 'Grande Pink Blush' as well as buds and flowers of Gaura lindheimeri 'Corinas Choice'(means for two years)

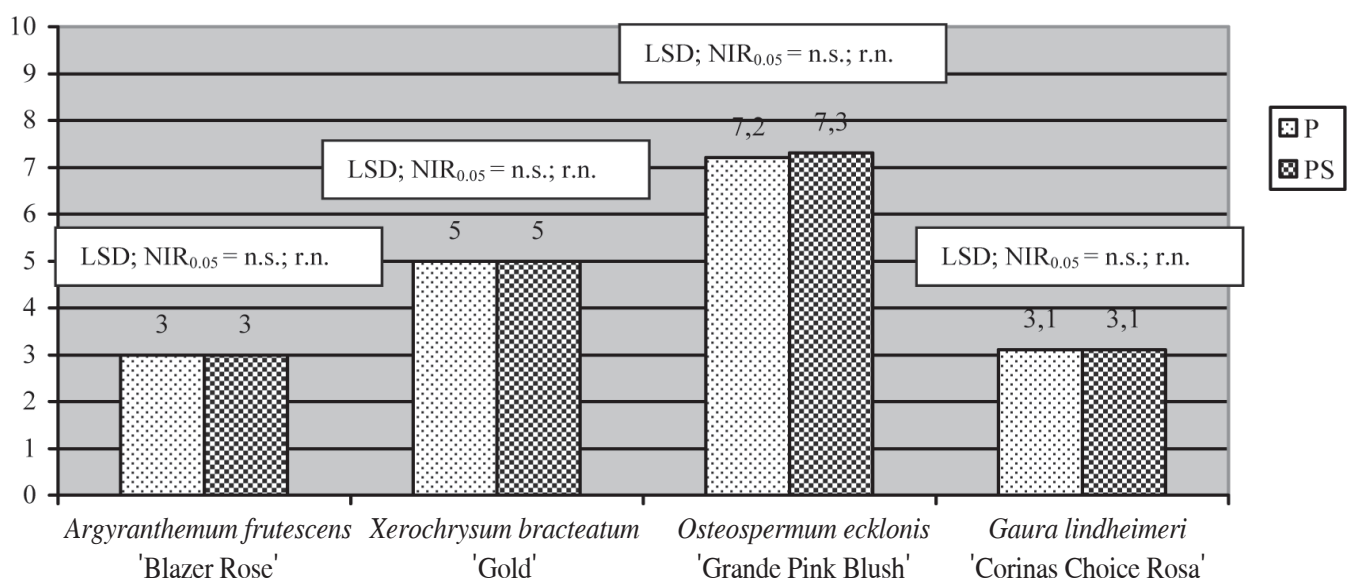

Fig. 3. The effect of type of medium on the diameter of inflorescences of Argyranthemum frutescens 'Blazer Rose', Xerochrysum bracteatum 'Gold' and Osteospermum ecklonis 'Grande Pink Blush' as well as flowers of Gaura lindheimeri 'Corinas Choice' (means for two years) [cm]

Legend: P means peat substrate, PS means peat substrate with sand in 3:1 ratio 


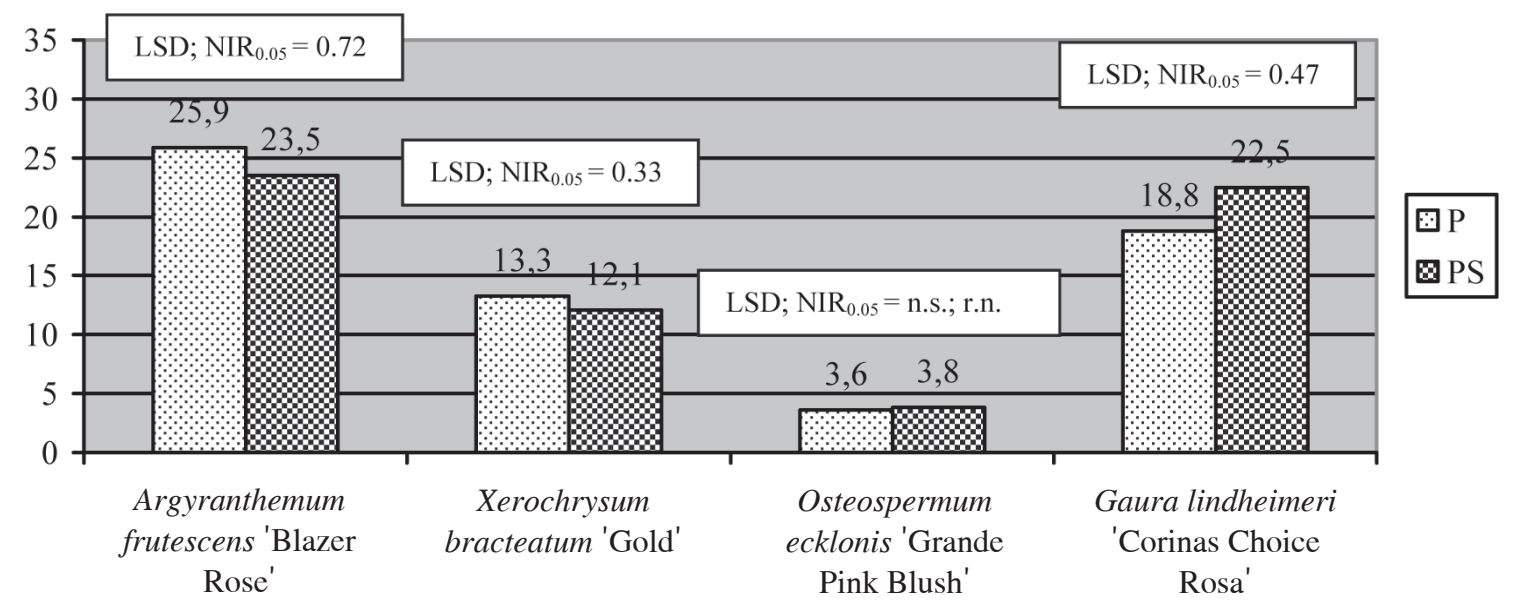

Fig. 4. The effect of type of medium on the number of buds and inflorescences of Argyranthemum frutescens 'Blazer Rose', Xerochrysum bracteatum 'Gold', and Osteospermum ecklonis 'Grande Pink Blush' as well as buds and flowers of Gaura lindheimeri 'Corinas Choice'(means for two years)

Legend: P means peat substrate, PS means peat substrate with sand in 3:1 ratio

\section{CONCLUSIONS}

1. Orthosilicic acid, contained in Actisil preparation, had a beneficial effect on the number of lateral shoots in all the examined plant varieties. In Argyranthemum frutescens 'Blazer Rose' and Xerochrysum bracteatum 'Gold', the number of shoots was higher according to the increase in concentration of fertilizing preparation.

2. Foliar application of Actisil positively influenced all characteristics of Xerochrysum bracteatum 'Gold'.

3 . The highest number of buds and flowers or inflorescences was recorded in ornamental plants of Xerochrysum bracteatum 'Gold' and Osteospermum ecklonis 'Grande Pink Blush' sprayed with Actisil at a concentration of $0.3 \%$, and $0.2 \%$ in the case of Argyranthemum frutescens 'Blazer Rose' and Gaura lindheimeri 'Corinas Choice'.

4. The effect of medium type on plant growth was related to plant species. Inhibited growth was shown by Argyranthemum frutescens 'Blazer Rose' and Osteospermum ecklonis 'Grande Pink Blush' grown in the medium with the addition of sand, although medium type did not affect the vegetative growth of Xerochrysum bracteatum 'Gold'.

\section{REFERENCES}

Carvalho M.P., Zanăo L.A. Jr., Grossi J.A.S., Barbosa J.G., 2009: Silicon improves ornamental pot sunflower production and quality. Cienc. Rural. 39 (8): 2394-2399, (in Spanish)

Epstein E., 2009: Silicon: its manifold roles in plants. Ann. Appl. Biol. 155: 155-160.

Frantz J.M., Pitchay S.D.S., Locke J.C., Horst L.E., Krause C.R., 2005: Silicon is deposited in leaves of New Guinea impatiens. Online. Plant Health Progress doi: 10.1094/PHP-2005-0217-01-RS.

Gillman J.H., Zlesak D.C., and Smith J.A., 2003: Applications of potassium silicate decrease black spot infection in Rosa hybrida 'Meipelta' (Fuschia Meidland). Hort Sci. 38: 1144-1147.

Hanafy Ahmed A.H., Harb E.E., Higazy M.A., Morgan Sh.H., 2008: Effect of silicon and boron foliar application on wheat plants grown under saline soil conditions. Int. J. Agri. Res. 3 (1): 1-26.

Hetman J., Maliborska M., Iwanow T., 2007: Wpływ Actisilu i sposobu uprawy na wzrost róży wielkokwiatowej Rosa multiflora. XI Ogólnopolska Konferencja Szkółkarska: Problemy i perspektywy produkcji szkółkarskiej roślin ozdobnych, ISiK- Skierniewice: 53-65 (in Polish).

Kamenidou S., Cavins T.J., Marek S., 2008: Silicon supplements affect horticultural traits of greenhouse-produced ornamental sunflowers. Hort Sci. 43 (1): 236-239.

Kamenidou S., Cavins T.J., Marek S., 2009: Evaluation of silicon as a nutritional supplement for greenhouse zinnia production. Sci. Hort. 119: 297-301.

Kamenidou S., Cavins T.J., Marek S., 2010: Silicon supplements affect floricultural quality traits and elemental nutrient concentrations of greenhouse produced gerbera. Sci. Hort. 123: 390-394.

Ma J.F., Yamaji N., 2008. Functions and transport of silicon in plants. Cell. Mol. Life Sci. 65: 3049-3057.

Marschner H., Oberle H., Cakmak I., Romheld V., 1990: Growth enhancement by silicon in cucumber (Cucumis sativus) plants depends on imbalance in phosphorus and zinc supply. Plant Soil. 124: 211-219.

McAvoy R.J., Bible B.B., 1996: Silica sprays reduce the incidence and severity of bract necrosis in poinsettia. Hort Sci. 31 (7): 1146-1149. 
Mikiciuk G., Mikiciuk M., 2009: Wpływ dolistnego nawożenia potasowo-krzemowego na wybrane cechy fizjologiczne truskawki (Fragaria ananassa Duch.) odmiany Elvira. / The influence of foliar application of potassium and silicon fertilizer on some physiological features of strawberry (Fragaria ananassa Duch.), variety Elvira. Ann. UMCS Sect. E 64 (4): 19-27 (in Polish).

Reynolds O.L., Keeping M.G., Meyer J.H., 2009: Silicon-augmented resistance of plants to herbivorous insects: a review. Ann. Appl. Biol. 155: 171-186.

Richmond K.E., Sus sman M., 2003: Got silicon? The non-essential beneficial plant nutrient. Current Opinion in Plant Biol. 6: 268-272.

$\mathrm{S}$ a c a 1 a E., 2009: Role of silicon in plant resistance to water stress. J. Elementol. 14(3): 619-630.

Savvas, D., Manos, G., Kotsiras, A., Souvaliot i s, S ., 2002: Effects of silicon and nutrient-induced salinity on yield, flower quality and nutrient uptake of gerbera grown in a closed hydroponic system. J. Appl. Bot. 76, 153-158.

Startek L., Placek M., Wraga K., 2006: Wpływ preparatu Hydro Plus Actisil na niektóre cechy chryzantem uprawianych w doniczkach. / The influence of HydroPlus ActiSil on some traits of pot chrysanthemum cultivars. Zesz. Probl. Post. Nauk Rol. 510: 619-626 (in Polish).

Wraga K., Dobrowolska D., 2007: Ocena wpływu preparatu Actisil na cechy morfologiczne i wartość dekoracją rozsad dwóch odmian bratka ogrodowego z grupy Fancy. Część I. Wzrost roślin i wielkość liści. / The estimation of effect of Actisil on morphological traits and decorative value of seedlings two garden pansy cultivars from Pansy gruops. Part. I. Plants growth and leaves size. Rocz. AR Pozn. CCCLXXXIII, Ogrodn. 41: 229-233 (in Polish).

\section{Wpływ dolistnego traktowania krzemem na rozwój sezonowych roślin ozdobnych. Część II: srebrzeń krzewiasty Argyranthemum frutescens 'Blazer Rose', kocanki ogrodowe Xerochrysum bracteatum 'Gold', osteospermum Ecklona Osteospermum ecklonis 'Grande Pink Blush' i gaura Lindheimera Gaura lindheimeri 'Corinas Choice'}

\section{Streszczenie}

Prowadzone w ostatnich latach badania potwierdziły, że nawożenie krzemem, wpływającym na odporność roślin na wiele czynników stresowych, pozytywnie oddziałuje na rozwój roślin, poprawiając jakość roślin ozdobnych. Dwuczynnikowe doświadczenie z czterema taksonami roślin sezonowych: srebrzeniem krzewiastym Argyranthemum frutescens 'Blazer Rose', kocankami ogrodowymi Xerochrysum bracteatum 'Gold', osteospermum Ecklona Osteospermum ecklonis 'Grande Pink Blush' i gaurą Lindheimera Gaura lindheimeri 'Corinas Choice' przeprowadzono w latach 2009-2010. Pierwszym czynnikiem doświadczenia było stężenie preparatu Actisil odpowiadające 60, 120 i $180 \mathrm{mg} \mathrm{Sixdm}^{-3}$ roztworu, aplikowanego trzykrotnie w formie oprysku, drugim typ podłoża: substrat torfowy oraz substrat torfowy $\mathrm{z}$ piaskiem $\mathrm{w}$ proporcji 3:1. Actisil wpłynął korzystnie na liczbę pędów bocznych wszystkich badanych w doświadczeniu odmian roślin. Największą liczbę pąków i kwiatów lub kwiatostanów stwierdzono u roślin Xerochrysum i Osteospermum ecklonis 'Grande Pink Blush' opryskiwanych Actisilem w stężeniu $0.3 \%$ oraz $0.2 \%$ u Argyranthemum i Gaura lindheimeri 'Corinas Choice'. Wpływ typu podłoża na wzrost roślin zależał od gatunku rośliny. U srebrzenia i osteospermum słabszym wzrostem odznaczały się rośliny uprawiane w podłożu z dodatkiem piasku, typ podłoża nie miał natomiast wpływu na rozwój wegetatywny kocanek ogrodowych. 